\title{
Energy Balancing of a Heuristic Algorithm for the Path Planning of Mobile Sensor Nodes
}

\author{
http://dx.doi.org/10.3991/ijoe.v12i05.5739 \\ X. Xu and B. Z. Liu \\ Chongqing College Of Electronic Engineering, Chongqing, China
}

\begin{abstract}
A wireless sensor network (WSN) is one of the core technologies of the Internet of things. It is an important means to realize a real-time geographic information system. Related research has shown that in the future, tens of billions of sensors and intelligent terminal equipment will be connected to WSNs based on the establishment of the function of the Internet of things. This study presents a heuristic algorithm to balance the energy consumption of each sensor node. It proposes a new real-time dynamic allocation algorithm for sensor tasks based on the concept of this heuristic algorithm and by considering that a multisensory system is composed of a phased-array radar. This allocation algorithm can dynamically assign tasks to the most suitable sensor before tasks fail to arrive, which ensures that the sensor can achieve a good load balance and extend network lifetime. A simulation experiment is conducted, and results validate the proposed algorithm. The energy consumption of mobile sensor nodes is effectively balanced. The pathplanning algorithm standardizes the energy consumption of each mobile sensor node across the network, thereby effectively prolonging network lifetime.
\end{abstract}

Index Terms-Function semantic clustering, Mobile sensor scheduling, Real-time GIS

\section{INTRODUCTION}

In a mixed wireless sensor network (WSN), static sensor nodes are distributed in the environment to collect data, and mobile sensor nodes within a certain region collect data in accordance with the planning of the line mobile. The mobile sensor nodes along a lattice network stop moving back and forth among stopping points, i.e., the center point of each cell. When mobile sensor nodes stop, the lattice network will bear all the data transmission work of the static sensor. The annual urban expansion area index, center of gravity transfer model, fractal dimension index, and elastic coefficient of urban expansionpopulation growth are used to explore the temporal and spatial characteristics of urban expansion [1]. The management of the Hanshiqiao Wetland Nature Reserve must focus on future changes in the wetland for its effective conservation [2]. The research results show that highcoverage grass and marsh areas decrease, low-coverage grassland increases, and the local eco-environment degenerates [3]. Meanwhile, renewable energy resources (RESs) are not fully environmentally safe. Different RESs are associated with varying environmental impacts [4]. The understanding of the spatial and temporal dynamics of the urban expansion of Hurghada is the cornerstone for formulating a view regarding future urban uses and for utilizing limited available resources [5].

\section{STATE OF THE ART}

The three foundations of modern information technology are sensor technology, communication technology, and computer technology, which realizes the collection, transmission, and processing of information, respectively. A sensor network combines these three technologies to achieve real unity of information collection, transmission, and processing. The sensor network is considered one of the most important technologies in the 21 st century; it is predicted to have a profound effect on the lifestyle of people in the future. With the development of wireless communication, microprocessor, and microelectromechanical system in recent years, sensor network technology has gradually matured, and its application has increasingly broadened. China has made numerous research achievements regarding sensor networks; however, they remain at the beginning stage. Such achievements have considerable strategic significance to the entire national economy and the social development of sensor network technology.

WSNs are small and low cost; they exhibit wireless communication, sensor, and data-processing capabilities in the work area. Each node may have different perceptual modalities, such as sonar, vibration wave, and infrared. Nodes can perform target information collection, transmission, and decision making for formulation and implementation to achieve regional monitoring, target-tracking, location, and prediction tasks. Each node can store, process, and transmit data. Sensor nodes cannot only exchange information with one another through a wireless network, but can also send messages to a remote terminal. WSNs are widely used in railways, smart homes, construction, agriculture, and so on.

\section{METHODOLOGY}

\section{A. Network Grid}

WSNs, which comprise microelectronics, sensors, communication, network, embedded systems, intelligent computing, and other technical fields, require multidisciplinary knowledge and high integration of the leading edges of various research fields. WSNs generally consist of a large number of sensor nodes deployed within the detection region. They implement dynamic and intelligent perception, acquisition, transmission, processing, and control of the network coverage area within the physical world of information in a collaborative manner. Figure 2 shows the system architecture of WSNs. The communication distance among sensor nodes is short, and the multihop method is generally used to realize long-distance data 

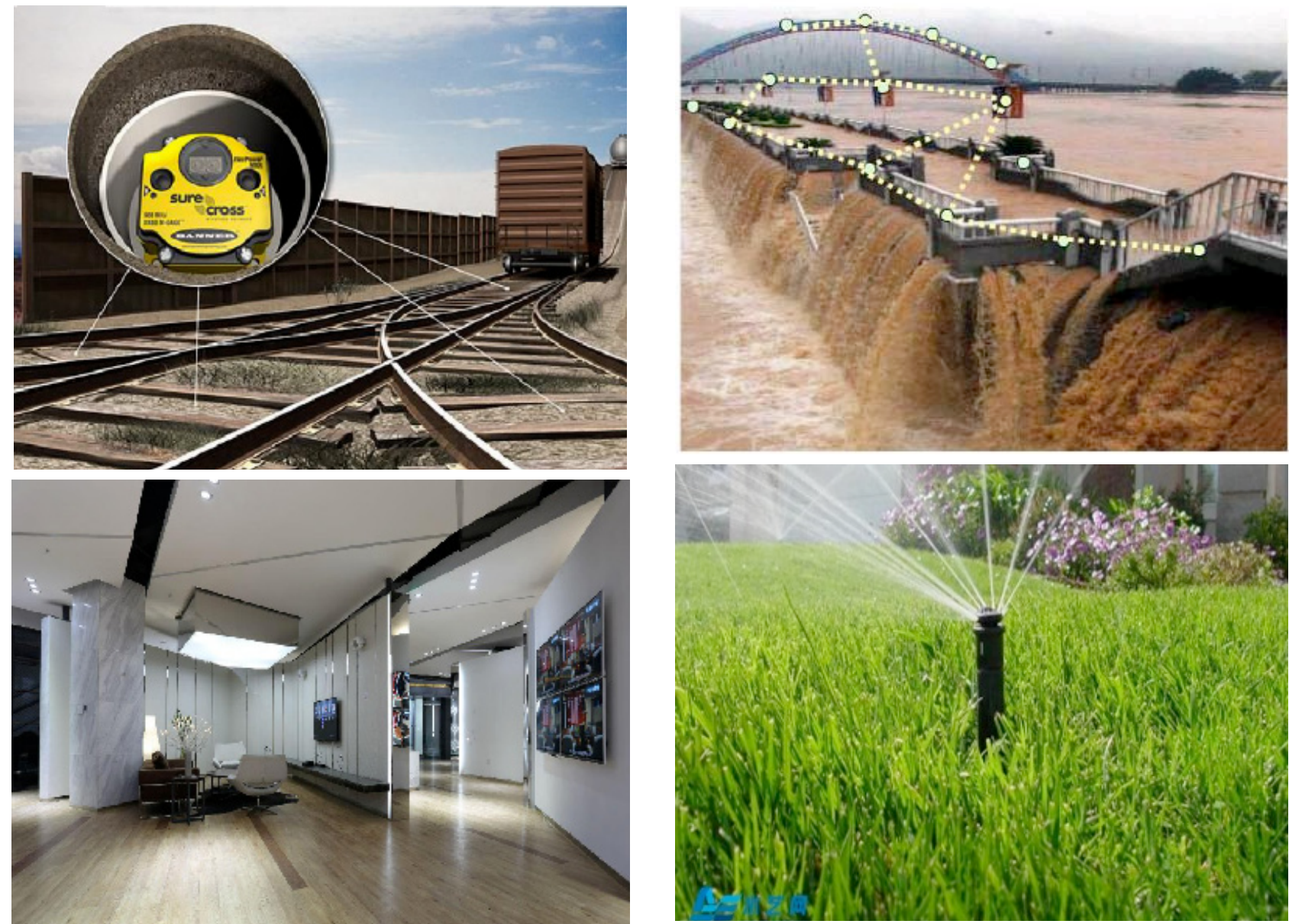

Figure 1. Application of WSNs in railways, smart homes, building inspection, and agriculture

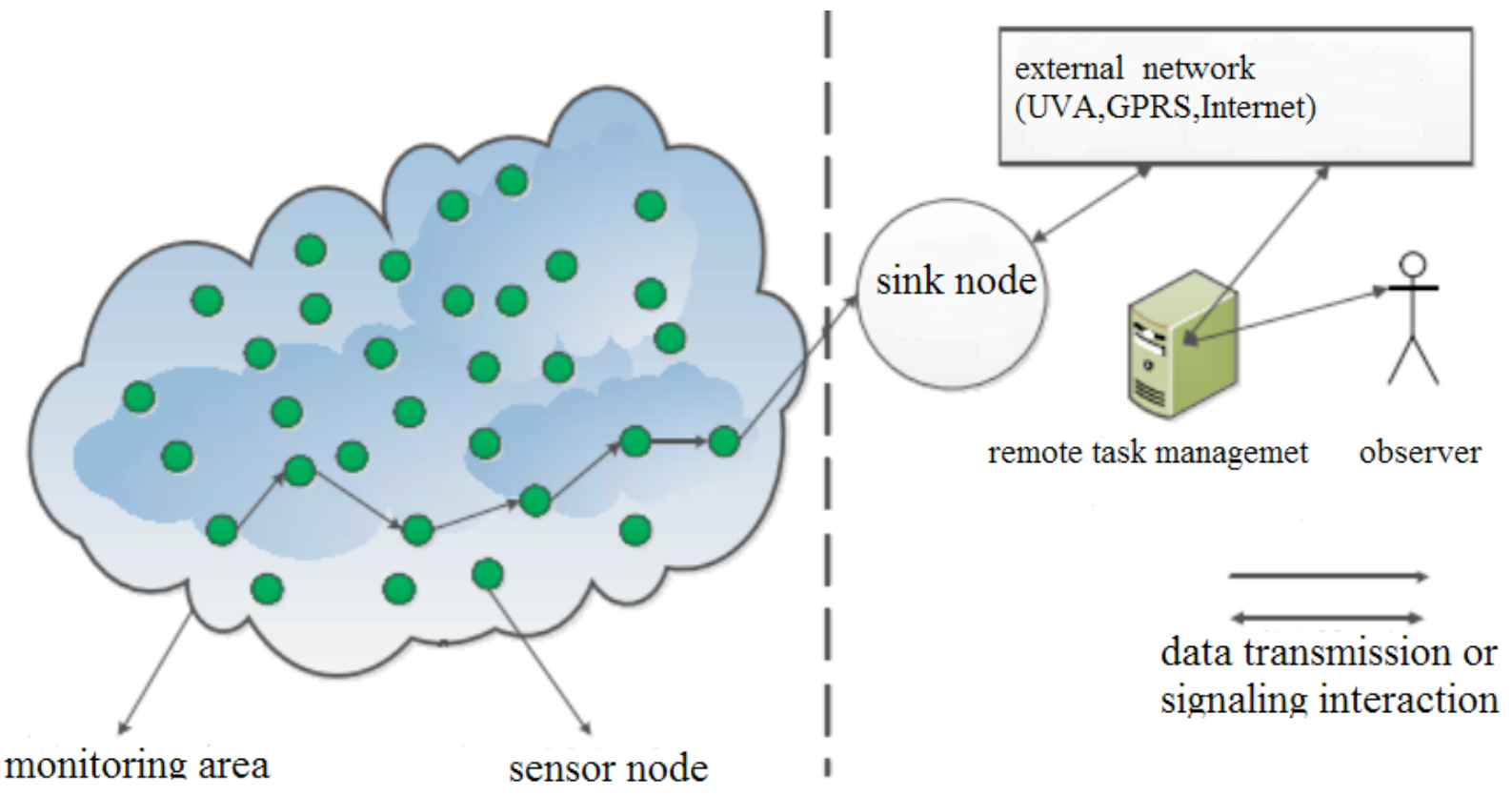

Figure 2. System architecture of WSNs

transmission [6]. Extensive convergence communication and data-processing capabilities are required to connect to a sensor network and an external network. A communication protocol between two protocol stacks is required to achieve conversion; remote task management instructions are forwarded to sensor nodes, the sensor nodes collect data, and the monitored event information is transmitted to a remote task management end [7].

Sensor nodes are generally powered by batteries with limited energy and are typically arranged in a complex environment; some areas are hardly reached by personnel. 
Consequently, replacing the battery is unrealistic and uneconomical for a large number of sensor nodes. The monitored data of sensor nodes via multihop relay are transmitted to the user via a sink link. Sensor nodes near sinks perform load data flow and experience premature death. The target frequently moves in the monitoring area, which causes some nodes close to the target to consume a considerable amount of energy, whereas most of the other nodes have sufficient remaining energy. This condition leads to network disconnection, energy hole, and information loss. Therefore, a prerequisite for reliably completing the target-tracking task is to maximize energy balance, and prolonging the network lifetime of sensor nodes is the key problem of target tracking in WSNs.

\section{B. Region-Partitioning Algorithm}

In this study, a hybrid WSN lattice is introduced after data transmission to maintain energy balance for the region division method. Unlike the $\mathrm{K}-\mathrm{D}$ tree-generation algorithm, the proposed algorithm checks units, and the area is in accordance with the generation from bottom to top and from left to right, namely, the $\mathrm{X}$-axis and $\mathrm{Y}$-axis coordinate value sequence. The region division algorithm is as follows.

The variance values of all the static sensor nodes are calculated, i.e., the $\mathrm{X}$-axis and $\mathrm{Y}$-axis variance values. The variance explains widely distributed static sensor nodes in the axis; thus, it should be perpendicular to the axis of the regional division. When the variance of the $\mathrm{X}$-axis at a large area is perpendicular to the divided X-axis, the static sensor nodes are arranged in ascending order of size according to their X-axis. In this sequence, the set of static sensor nodes in e to Sn on the target node, e.g., when e = 3.34 and $\mathrm{e}=4$, is in a sequence of fourth nodes for the target node. The target node in which the lattice is perpendicular to the $\mathrm{X}$-axis is two. If the target node is on the left side of a partition, then the generation area does not include the target node in the grid; if the target node is on the right side of the partition, then the generation area includes the target node in the grid. Accordingly, the new generation area contains a number of static sensor nodes, including those in the ideal areas. When the variance of the $\mathrm{Y}$-axis is large, the division process is reversed. The new area in the lattice is removed from the entire region, whereas the rest of the grid is divided according to the preceding algorithm until the hybrid WSN is divided into $\mathrm{m}$ regions. The algorithm complexity is $\mathrm{O}(\mathrm{n})$, and $\mathrm{m}$ is in the termination region of the algorithm. The number of static sensor nodes in the $\mathrm{m}$ region is approximately equal, i.e., the algorithm of the mobile sensor nodes in each region is approximately equal to the energy consumption of data transmission.

The grid-computing method is widely used in WSNs. This section mainly introduces the heuristic energy balance of mobile sensor nodes for the path-planning algorithm, which is the first step of the lattice. The main purpose of the grid-computing method is to establish a suitable grid for the subsequent steps. Energy balancing and path planning are performed for the succeeding steps by simplifying the calculation process of the direct target from the static sensor nodes to the grid. The advantage of the lattice is that it can simplify target computation; moreover, it uses lattice as the basic unit to calculate energy load and moving path. The utility model also has the following advantages. First, having lattice as the basic unit can avoid the influence of single static sensor nodes from the network on the path-planning result. Second, the pathplanning algorithm overcomes considerable complication.

Figure 3 presents examples of partitioning a region. For a brief description of the division process, Figure 4 shows the hybrid WSN scheme that is divided into three regions. The scheme contains a total of 10 static sensor nodes, namely, $\mathrm{n}_{1}(1,1), \mathrm{n}_{2}(1,5), \mathrm{n}_{3}(3,3), \mathrm{n}_{4}(3,9), \mathrm{n}_{5}(5,1), \mathrm{n}_{6}$ $(5,9), \mathrm{n}_{7}(7,3), \mathrm{n}_{8}(7,5), \mathrm{n}_{9}(9,5)$, and $\mathrm{n}_{10}(9,9)$. In this example, the grid side is gSide $=2$ per unit length, $m=3$, and $\mathrm{e}=3.34$. In the first iteration, all the 10 nodes of the $\mathrm{X}$-axis have coordinate values of variance vex $=8$, whereas those of the Y-axis have coordinate values of variance vie $=8.8$; thus, the first region will be perpendicular to the $\mathrm{Y}$-axis division. All the static sensor nodes are sorted from small to large in accordance with their $\mathrm{Y}$-axis coordinate values. The fourth position in the static sensor nodes for the target node is son $=\mathrm{n} 7$ given that $\mathrm{e}=4$. In Figure 4 , Tbottom Ttop has distance N7 from the two recent lattice fields, and then $|\mathrm{c} \mathrm{T}-\mathrm{e}|=0.66$ and $\mathrm{B}|\mathrm{c}-\mathrm{e}|=1.34$ are calculated. Consequently, Top is used as the boundary of the region to make the static sensor nodes in the region close to the ideal condition. The red area in Figure 4 is the first area S1, which is the final partition. S1 deletes from the network and continues to divide the remaining grid [8].

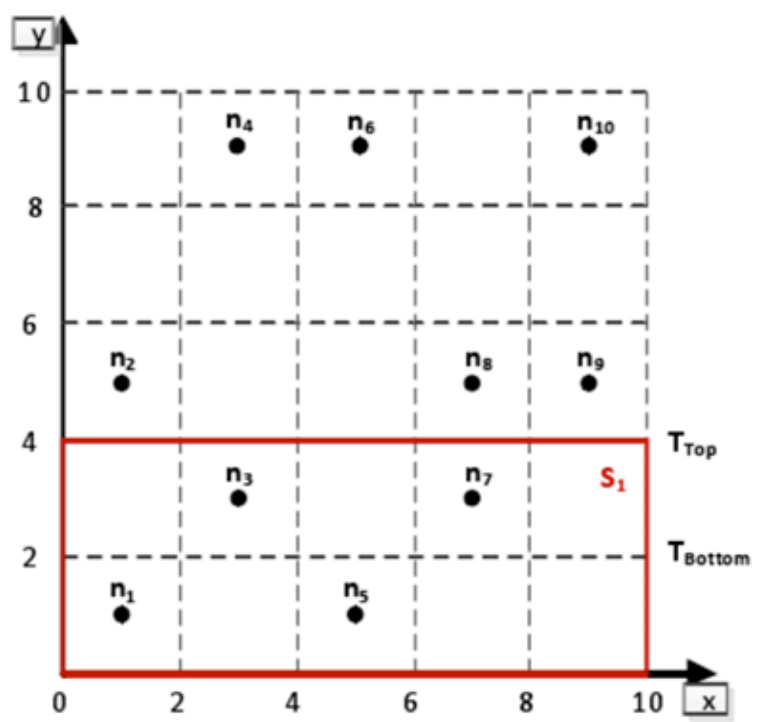

Figure 3. Example of the region-partitioning algorithm

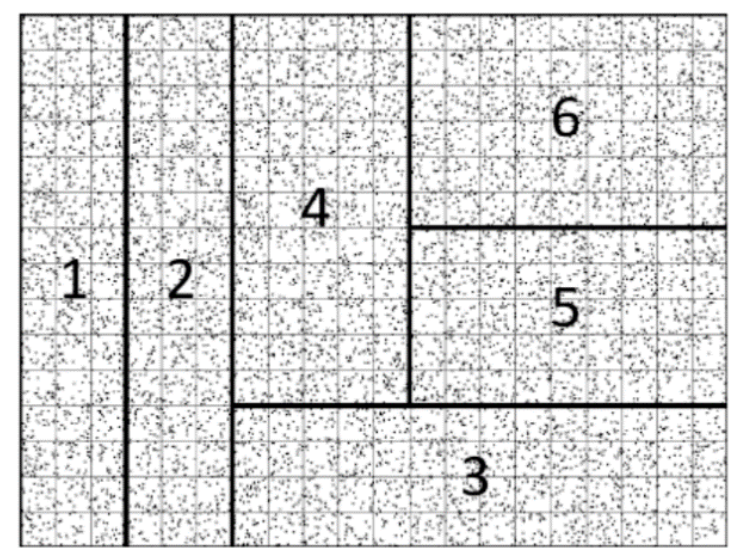

Figure 4. Chart of the experimental results after implementing the region-partitioning algorithm 
The experimental results for the region division algorithm are shown in Figure 4. The black origin indicates the simulation of the static sensor nodes, the black lines denote the boundary lattice, and the heavy black line represents the boundary of the region. Numbers are generated in each region for the regional order, namely, from left to right and from bottom to top.

In implementing regional partitioning based on the lattice, roughly the same number of static sensor nodes exists in each region. Each mobile sensor node in the regional data of transmission energy consumption in this study should be approximately equal. The energy consumed during the physical movement of mobile sensors and during data transmission is considered under the condition of each region by transmission grid rebalancing, namely, mobile rebalancing of the energy consumption of sensor nodes.

The proposed regional balancing algorithm is a heuristic algorithm that adjusts generated numbers according to regions. When in a region, the total energy consumption of mobile sensor nodes (data transmission energy and total energy in the local physical movement) is large; this area should pass the appropriate neighbor grid area. When considering local time, the need from neighborhood right will transfer back some of the lattices. Regional adjustment is reconducted after a round of adjustment to determine whether the $M$ energy consumption of the variance termination condition of the mobile sensor node is satisfied after the end of the round of adjustment. If the condition is satisfied, then the algorithm terminates; otherwise, the adjustment is continued.

In this algorithm, the stable state is defined as follows: the $\mathrm{V}$ algorithm of $\mathrm{V}$ is unchanged or enters a steady-state cycle within three iterations. V entering into the circulation indicates the presence of several lattices in the area among the repeated transfer activities, which shows that the energy consumption of mobile sensor nodes is balanced under the current condition. When $\mathrm{V}$ is unstable, the adjustment of each region is according to the build order area, and thus, the regional grid judgment conditions should be reduce or increase based on the energy consumption for mobile sensor nodes. According to the criteria, the region should reduce or increase the lattice. The complexity of this algorithm is $\mathrm{O}\left(\mathrm{n}^{2}\right)$.

Only the lattice close to the initial state region boundary can pass through during the delivery process in two regional grids. In the second step, the division results close to the lattice of dividing lines can be marked as GN (I, J); in the subsequent process, these areas belong to GN (I, J). The number of checks will not exceed the amount of the initial condition. Figure 6 presents an example; numbers $2,7,11$, and 15 belong to $\operatorname{GN}(2,1)$, whereas numbers 6 , 10 , and 14 belong to $\operatorname{GN}(1,2)$. Figure 5 illustrates the three grids (numbers 2, 6, and 10) transferring from region $\mathrm{C} 1$ to regions $\mathrm{C} 2$ and $\mathrm{GN}(\mathrm{I}, \mathrm{J})$, which are included in the change process.

Figure 6 shows the simulation results of the regional energy balance of the map. The origin is shown in the black static sensor nodes, black wire grid boundary, and the thick black region boundary. Each region is within the digital regional generated order. The entire boundary has many irregular protrusions. The irregular boundary will affect the physical movement of mobile sensor nodes to traverse the entire region, and thus, increase the energy consumption to a certain extent.
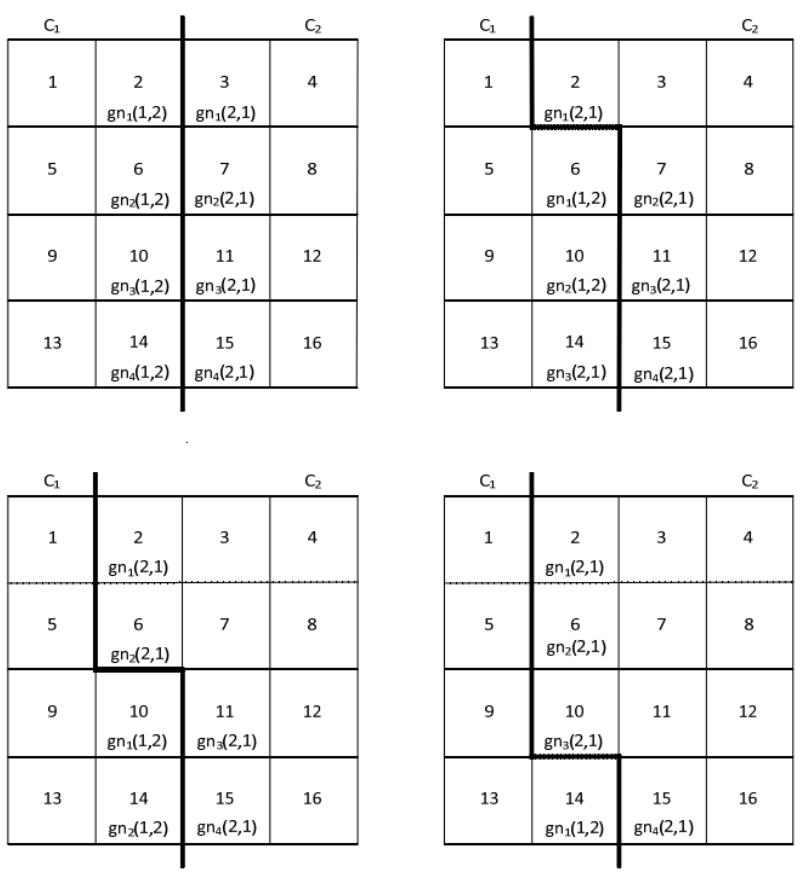

Figure 5. Transfer from C1 to the regional grid area of C2 GN (I, J) that is included in the change process

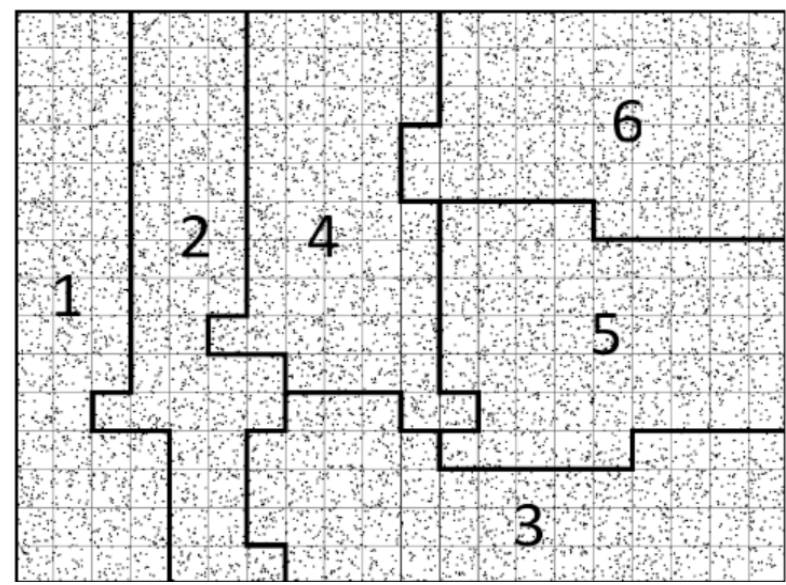

Figure 6. Experimental results of regional rebalancing

\section{RESUlt ANALYSIS AND Discussion}

\section{A. Simulation Experiment}

The simulation test program algorithm realizes a program with Java 6 mobile sensor nodes and 10000 static sensor nodes. The $1000 \mathrm{~m} \times 750$ simulated hybrid WSN is distributed randomly in space. This study also sets up $\mathrm{R}=$ $7543130=50 \mathrm{~m}$, which enables the mobile nodes in a grid station to complete data transmission in a wireless sensornetwork and to jump the distance of static sensor nodes. The hybrid grid has $20 \times 15=300$ lattice. A mobile sensor is used in the hybrid WSN simulation. The moving finite element assumes that one energy transmission distance is 20 times that of static sensor energy consumption, which can be in proportion to changes under different situations. The experiment simulates seven sets of experimental data.

\section{B. Experimental Result}

Figure 7 shows the seven sets of experimental data; the $\mathrm{X}$-axis presents the iterative times, whereas the $\mathrm{Y}$-axis 
denotes the energy consumption variance for each mobile sensor. This figure reflects the degree of energy balance of each mobile sensor, which implies the effective energy balance of the path-planning algorithm proposed in this study. As shown in the results in Figure 7, the energy consumption of each sensor is uneven before the start of the algorithm, i.e., the variance is considerable. After the algorithm is implemented up to the fourth iteration, the situation is considerably improved; the Y-axis is adjusted to obtain the result presented in Figure 8 [9].

In this figure, the iterative algorithm for approximately 12 times to 14 times can be in a stable state. The figure also shows that the $\mathrm{V}$ values likely swing for a certain period before achieving a stable state. Some of the lattices repeatedly transfer and have large $\mathrm{V}$ values. The lattices transfer toward the end of the algorithm. When $\mathrm{V}$ values become stable, the algorithm at the end of the hybrid WSN has a minimum value of the lattice iteration of the regional division. In each regional planning, mobile sensor nodes present a traversal path diameter, namely, along the center point of a lattice that is moving to complete data transmission of the region [10].

Figures 7 and 8 show that the irregular boundary will cause the mobile sensor node to consume the moving distance of additional complete regional data collection work. However, many irregular results are presented in Figures 7 and 8 . The irregular boundary will consume additional energy in the actual mobile sensor node in the application, and thus, cause large errors between the experimental results and the practical application of the algorithm in this study.

After the improvement, the regional rebalancing experimental results shown in Figure 9 indicate that the boundary of each region relative to Figure 6 does not improve, which is conducive to the movement of mobile sensor nodes in area efficiency. The algorithm error and the practical application decrease. From Figures 10 and 11, after the rebalancing algorithm in the lattice exchange sequence is improved, the effectiveness of the algorithm is not affected. The algorithm starts with a large variance. After a certain number of iterations, $\mathrm{V}$ becomes stable.

As the region of the lowest point of $\mathrm{V}$ on each line divides, the lowest point of each data set represents the algorithm result. As shown in Figures 8 and 11, in addition to data set 2 , the other data sets in the improved algorithm present decreased $\mathrm{V}$ values. Therefore, lattice order exchange is improved in the $\mathrm{uECM}=20 \times$ gi.ECD design algorithm. The algorithm effect is more evident in the application of the energy consumption of physical movement than in the energy consumption of data transmission.

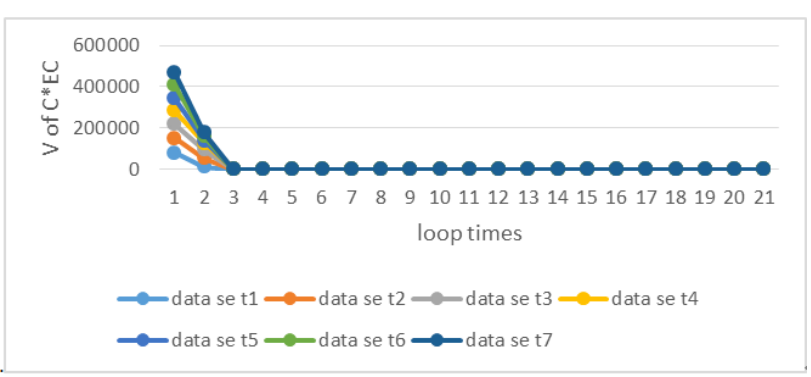

Figure 7. Simulation experiment test data on mobile sensor energy consumption variance results 1

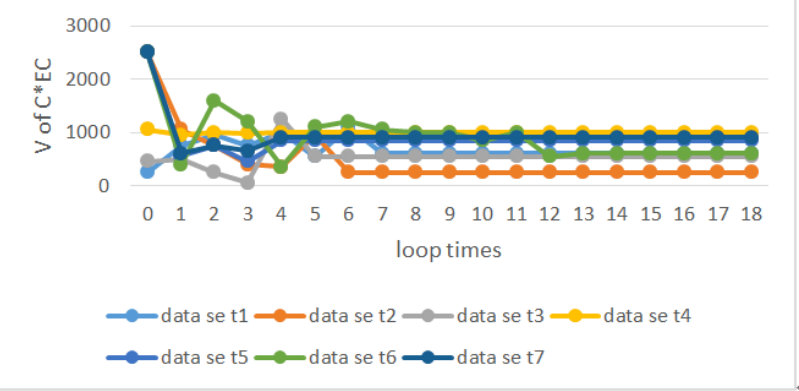

Figure 8. Simulation experiment test data on mobile sensor energy consumption variance results 2

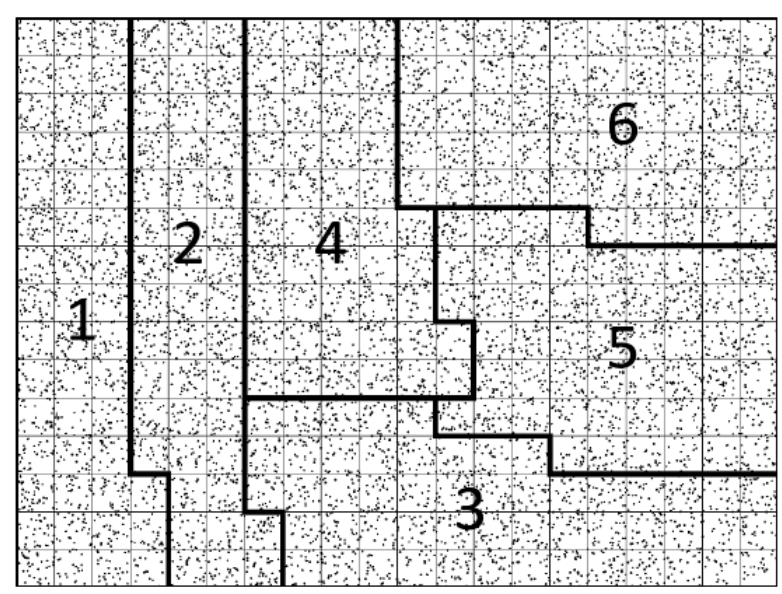

Figure 9. Experimental results of the improved region rebalancing

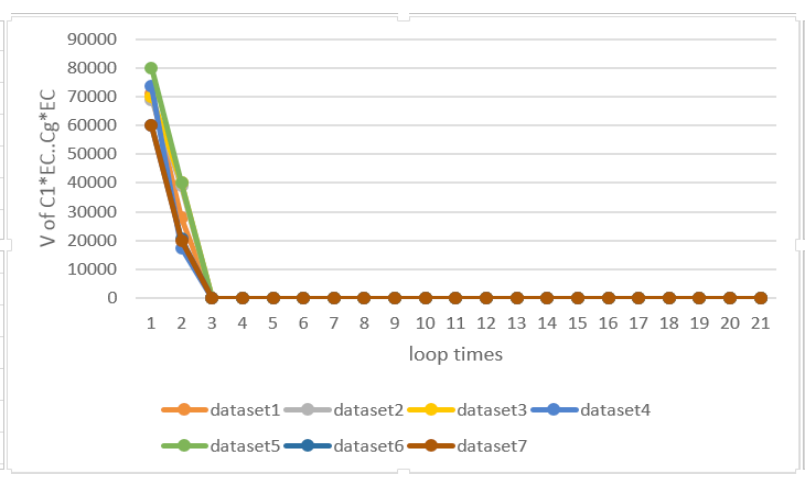

Figure 10. Simulation experiment test data on mobile sensor energy consumption variance results 1

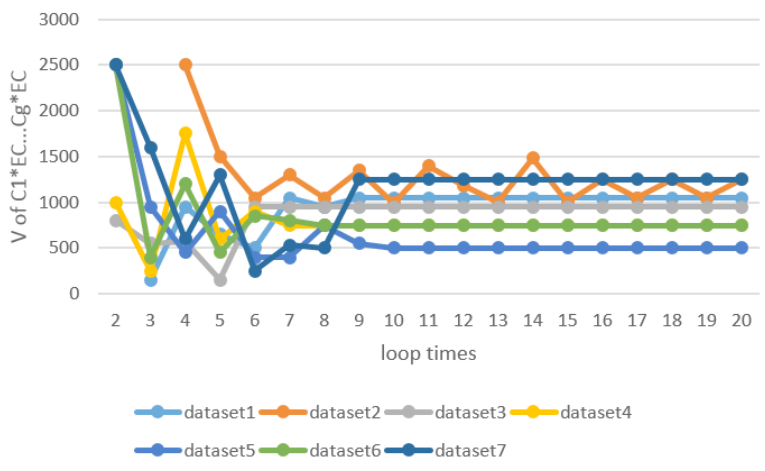

Figure 11. Simulation experiment test data on mobile sensor energy consumption variance results 2 


\section{CONCLUSION}

This study proposes a heuristic algorithm to balance the energy consumption of each sensor. The algorithm can dynamically assign tasks to the most appropriate sensor before tasks fail to arrive, which ensures that the sensor can achieve good load balancing and prolong network lifetime.

The experimental results show that the proposed hybrid WSN energy balance path-planning algorithm is effective. The WSN node energy consumption and a single pathplanning algorithm of mobile sensor nodes compared with the algorithm pseudocode are introduced in detail. The algorithm implementation details and principles are also provided. The simulation experiment proves the validity of the proposed algorithm. This algorithm can be applied to large-scale network scenes; it considers mobile sensor nodes based on an event-driven programming algorithm to solve the problem of large-scale data acquisition. This algorithm can also freely control regional data, i.e., whether WSN nodes join or leave a network, to replan the path of each mobile sensor node. The implementation conforms to practical application. Therefore, the proposed heuristic energy balancing of the mobile sensor nodes of the path-planning algorithm can guarantee balanced energy consumption among hybrid WSN applications for mobile sensor nodes and prolong the working life of the network. After a static sensor is arranged, its physical location will not change. Accordingly, in case of constant parameters, the results of the second step region of the division algorithm are repeatable to optimize the results of irregular boundary problems and the provisions of regional exchange among lattice orders. The same rule applies to the third step.

\section{REFERENCES}

[1] Sakhaeifar, Alaleh, and S. Hadi Ghoddusifar. "Impact of Location-Behavior on Sense of Belonging to Place," Modern Applied Science, 2016, vol. 10, no. 5, pp. 57. http://dx.doi.org/10.5539/ mas.v10n5p57

[2] Takagi, Hiroshi, et al., "Ocean tide modelling for urban flood risk assessment in the Mekong Delta," Hydrological Research Letters, 2016, vol. 10, no.1, pp. 21-26. http://dx.doi.org/10.3178/hrl.10.21
[3] Garau, Chiara, Francesca Masala, and Francesco Pinna, "Cagliari and smart urban mobility: Analysis and comparison," Cities, 2016, vol. 56, pp. 35-46. http://dx.doi.org/10.1016/j.cities.2016.02.012

[4] Vutete, Clever, and Farai Chigora, "The Rural Market and Urban Market Integration: A Marketing Panacea to Economic Development Issues of Zimbabwe," Business and Economic Research, 2016, vol. 6, no.1, pp. 234-247. http://dx.doi.org/10.5296/ ber.v6i1.9168

[5] Salmond, Jennifer A., et al., "Health and climate related ecosystem services provided by street trees in the urban environment," Environmental Health, 2016, vol. 15, no.1, pp. 95. http://dx.doi.org/10.1186/s12940-016-0103-6

[6] Devi, U. Kavitha, and V. Vasanthakumari., "Iron Profile of Obese female adults in Sivaganga District," World Journal of Science and Research, 2016, vol. 1, no.1, pp. 06-11.

[7] Papadopoulou, Maria, et al., "Evaluating predictive performance of sensor configurations in wind studies around buildings," $\mathrm{Ad}$ vanced Engineering Informatics, 2016, vol. 30, no.2, pp. 127-142. http://dx.doi.org/10.1016/j.aei.2016.02.004

[8] Usanova, M. E., I. R. Mann, and F. Darrouzet., "EMIC Waves in the Inner Magnetosphere," Washington DC American Geophysical Union Geophysical Monograph Series, 2016, vol. 216, pp. 65-78. http://dx.doi.org/10.1002/9781119055006.ch5

[9] Manuel, Sandra, "Sexuality and Gender Politics in Mozambique: rethinking gender in Africa by Signe Arnfred (review)," Africa: The Journal of the International African Institute, 2015, vol. 85, no.4, pp. 737-737. http://dx.doi.org/10.1017/S0001972015000741

[10] Rodger, Craig J., et al., "High resolution in situ observations of electron precipitation-causing EMIC waves," Geophysical Research Letters, 2015, vol. 42, no.22, pp. 9633-9641. http://dx.doi.org/10.1002/2015GL066581

\section{AUTHORS}

X. Xu is with the Chongqing College of Electronic Engineering, Chongqing, China (e-mail: 13500301132@163.com).

B. Z. Liu is with the Chongqing College of Electronic Engineering, Chongqing, China (e-mail: 13500301132@163.com)

This work is supported by the Chongqing Education Commission scientific research topic "Research on landslide monitoring and early warning system based on Wireless Sensor Network" the project number: kj132204. Submitted, February, 19, 2016. Published as resubmitted by the authors on May, 16, 2016. 